\title{
LA COLECCIÓN «ESTUDIS SOBRE LA TRADUCCIÓ» DE LA UNIVERSITAT JAUME I \\ Josep Marco \\ Universitat Jaume I
}

\section{ABSTRACT}

This article has two aims: a) to report on the origins of the series Estudis sobre la traducció, published by Publicacions de la Universitat Jaume I, and provide an account of its defining features, such as readership profile, languages used and kinds of works included (collective volumes, monographs, etc.); and b) to offer a list of the books making up the series and provide a very brief overview of each, including its structure and content.

KEY WORDS: Estudis sobre la traducció series, readership profile, kinds of books, overview of the books

\section{RESUMEN}

El presente artículo persigue dos objetivos: a) dar cuenta del origen de la colección «Estudis sobre la traducció», de Publicacions de la Universitat Jaume I, e informar sobre algunos de los rasgos que la definen, como el perfil del destinatario, las lenguas que se utilizan y los tipos de obras que incluye (volúmenes colectivos, monografías, etc.); y b) proporcionar un listado de los libros de que consta la colección y ofrecer una breve glosa de cada uno de ellos, en la que se presentan de modo muy sucinto su estructura y contenido.

PALABRAS CLAVE: colección «Estudis sobre la traducció», perfil del destinatario, tipos de obras, glosa de los libros

\section{ORIGEN DE LA COLECCIÓN Y RASGOS QUE LA DEFINEN}

La colección «Estudis sobre la traducció» nació de la mano de la implantación de la Licenciatura en Traducción e Interpretación en la Universitat Jaume I (UJI) en el año 1994. De hecho, estrictamente hablando, la primera precedió a la segunda por unos meses. En mayo de 1993 se celebraron las primeras jornadas sobre la traducción organizadas por la universidad castellonense, que giraron alrededor de la teoría de la traducción, y justo un año más tarde vio la luz el primer volumen de la colección, que recogía las 
contribuciones a dichas jornadas; en cambio, la licenciatura no echaría a andar hasta septiembre de ese mismo año, 1994 (aunque en 1992-93 tuvo lugar un curso de posgrado sobre traducción que podría considerarse la primera piedra de la licenciatura). Las jornadas de traducción de la UJI han tenido y tienen un formato singular, ya que presentan un programa cerrado, sin invitación abierta (call for papers) a la comunidad científica del campo de la traductología. Por lo tanto, ese primer volumen de la colección no era una selección de contribuciones, como ocurre con mucha frecuencia en otros encuentros científicos, sino un compendio de todas las conferencias pronunciadas en el curso de las jornadas.

La persona clave en los distintos desarrollos apuntados en el párrafo anterior (implantación de la licenciatura, creación de la colección, organización de las primeras jornadas) y aun de otros (como la creación de un grupo de investigación en traductología, $y$, más concretamente, en didáctica de la traducción) fue sin duda la profesora Amparo Hurtado Albir. A ella debemos el impulso inicial y la consolidación de todas estas iniciativas. Hoy en día la existencia de colecciones específicas de traducción e interpretación puede parecer a muchos algo natural, que no necesita mayores explicaciones porque se justifica por sí misma; sin embargo, no era éste el caso en 1994, cuando la mayor parte de monografías de nuestro campo se publicaban en colecciones de más amplio espectro. De hecho, la colección (quizá) más prestigiosa que existe actualmente en nuestra disciplina (la Benjamins Translation Library) data precisamente de ese año. Por lo tanto, se requería cierta dosis de visión y cierta voluntad de asumir riesgos para tomar un camino no muy frecuentado ni hollado.

Si, desde el principio mismo de este artículo, y a propósito de la colección que aquí nos ocupa, me he referido a las jornadas de traducción, es porque la relación entre ambas iniciativas no es banal ni pasajera, sino esencial y duradera. De hecho, una de las funciones primordiales que pretendía desempeñar la colección desde sus inicios era publicar las aportaciones de los especialistas de primer nivel que eran invitados a participar en dichas jornadas, al principio con una periodicidad anual y, a partir de su tercera edición, en 1995, cada dos años. Sin embargo, la colección quería nutrirse también de monografías, tanto de autores de la Universitat Jaume I como de otras procedencias. Era una colección abierta, sin prejuicios ni restricciones temáticas, que pretendía cubrir los distintos ámbitos de especialidad de la traductología así como sus principales cuestiones transversales. En este sentido, cabe decir que la colección habría podido ser la vía natural de publicación de las tesis doctorales que, a lo largo de la década de los 90 y en los primeros años del siglo XXI, iban elaborando y defendiendo los miembros del Departamento de Traducción y Comunicación de la UJI. Pero la devaluación de que con tanta 
frecuencia son objeto las publicaciones que ven la luz en la misma universidad en la que trabaja su autor en los procesos de reconocimiento de tramos de investigación y, más recientemente, de acreditación mermó en gran medida el potencial de la colección, ya que los autores de la casa optaron por publicar los resultados de sus tesis en otras editoriales. Es éste un buen ejemplo de cómo una línea de conducta bien intencionada -ya que la mencionada devaluación no pretendía sino evitar la endogamia en los circuitos de las publicaciones científicas- puede producir efectos perversos, pues la colección podría haberse nutrido de aportaciones de rigor y calidad que tuvieron que buscar otras salidas. Nuestra colección se resintió de ello sobre todo en términos cuantitativos, ya que se publicó menos de lo que se podría haber publicado.

Los destinatarios naturales de los volúmenes incluidos en esta colección son fundamentalmente los traductólogos, es decir, los investigadores y estudiosos que tienen como objeto de estudio la traducción y la interpretación. Desde aquel ya lejano 1994, es evidente que la masa crítica de dicho colectivo ha aumentado notablemente, por lo que el número de lectores potenciales es mucho mayor que entonces. En segundo lugar, cabe mencionar también a los estudiantes de traducción e interpretación. Si bien la colección no se dirige a ellos de modo primordial, ya que no tiene vocación didáctica ni incluye manuales prácticos, muchas de las contribuciones a las jornadas de traducción publicadas luego en forma de capítulo de libro pueden encontrar cabida perfectamente en el aula de traducción. Finalmente, también los traductores profesionales pueden beneficiarse de la lectura de estas publicaciones, que con frecuencia giran en torno a cuestiones que inciden muy directamente en la práctica de la traducción.

Las lenguas vebiculares, por así decirlo, de la colección son, por orden de importancia, el castellano, el catalán y el inglés. Si entendemos por lengua vebicular aquélla en la que se expresa el título y el prólogo o introducción, el castellano lo es en 13 de los 16 volúmenes que hasta la fecha componen la colección, mientras que el catalán lo es en 2 (los dos primeros) y el inglés en 1 (un caso absolutamente singular, que se explica por el hecho de haber sido cofinanciado por una universidad norteamericana). Sin embargo, hay mayor variedad en lo que respecta a las lenguas utilizadas en los volúmenes colectivos, puesto que, en la mayoría de los casos, éstos incluyen contribuciones o capítulos en español, catalán e inglés. También el francés está representado en varios volúmenes, y el italiano en uno. En cuanto a las monografías, las cuatro que incluye la colección están redactadas en español.

Como ya se ha comentado más arriba, la creadora y primera directora de la colección fue Amparo Hurtado Albir. Cuando la profesora Hurtado dejó la Universitat Jaume I para incorporarse a la Universitat Autònoma de Barcelona, la colección fue gestionada durante algunos años desde la dirección 
del Servicio de Publicaciones, hasta que en 1999 la universidad decidió crear un nuevo organigrama y nombrar directores para cada una de las colecciones de la casa. Desde 1999 hasta 2008, la colección fue dirigida por el profesor Josep Marco, quien fue reemplazado a finales de 2008 por el profesor Frederic Chaume, el director actual. En el capítulo de las personas que han contribuido a moldear la colección, hay que mencionar forzosamente a Carme Pinyana Garí, editora de Publicacions de la Universitat Jaume I, quien ha desempeñado un papel importantísimo en la recepción de originales, su adscripción a una u otra colección, la elección de evaluadores externos y el asesoramiento en la toma de decisiones por parte del director de la colección.

\section{LIBROS QUE COMPONEN LA COLECCIÓN Y BREVE GLOSA DE CADA UNO DE ELLOS}

La colección «Estudis sobre la traducció» consta hasta la fecha de los siguientes volúmenes:

1. Hurtado Albir, Amparo (ed.) (1994): Estudis sobre la traducció. Castelló: Publicacions de la Universitat Jaume I. ISBN: 84-8021-041-9.

2. Marco Borillo, Josep (ed.) (1995): La traducció literaria. Castelló: Publicacions de la Universitat Jaume I. ISBN: 84-8021-057-5

3. Hurtado Albir, Amparo (ed.) (1996): La enseñanza de la traducción. Castelló: Publicacions de la Universitat Jaume I. ISBN: 84-8021-078-8.

4. Blake, William (introducción, traducción, notas y glosario a cargo de Xavier Campos Vilanova) (1997): Jerusalén, la emanación del gigante Albión. Castelló: Publicacions de la Universitat Jaume I. ISBN: 978-84-8021-122-2.

5. García Izquierdo, Isabel y Joan Verdegal (eds.) (1998): Los estudios de traducción: un reto didáctico. Castelló: Publicacions de la Universitat Jaume I. ISBN: 84-8021-204-7.

6. Benet, Vicente J. y Eloísa Nos (eds.) (1999): Cuerpos en serie. Castelló: Publicacions de la Universitat Jaume I. ISBN: 978-84-8021-294-6.

7. Chaume, Frederic y Rosa Agost (eds.) (2001): La traducción en los medios audiovisuales. Castelló: Publicacions de la Universitat Jaume I. ISBN: 84-8021$321-3$.

8. Mayoral, Roberto (2001): Aspectos epistemológicos de la traducción. Castelló: Publicacions de la Universitat Jaume I. ISBN: 84-8021-345-0. 
9. Martínez Romero, Tomàs y Roxana Recio (eds.) (2001): Essays on Medieval Translation in the Iberian Peninsula. Castelló: Publicacions de la Universitat Jaume I y Creighton University. ISBN: 84-8021-365-5.

10. Alcina Caudet, Amparo y Silvia Gamero Pérez (eds.) (2002): La traducción cientifico-técnica y la terminología en la sociedad de la información. Castelló: Publicacions de la Universitat Jaume I. ISBN: 84-8021-409-0.

11. Grupo CRIT (2003): Claves para la comunicación intercultural. Castelló: Publicacions de la Universitat Jaume I. ISBN: 84-8021-430-9.

12. García de Toro, Cristina e Isabel García Izquierdo (eds.) (2005): Experiencias de traducción. Reflexiones desde la práctica traductora. Castelló: Publicacions de la Universitat Jaume I. ISBN: 84-8021-485-6.

13. Monzó, Esther y Anabel Borja (eds.) (2005): La traducción y la interpretación en las relaciones jurídicas internacionales. Castelló: Publicacions de la Universitat Jaume I. ISBN: 84-8021-540-2.

13. Molina, Lucía (2006): El otoño del pingüino. Análisis descriptivo de la traducción de los culturemas. Castelló: Publicacions de la Universitat Jaume I. ISBN: 84-8021-519-4.

15. Martínez Sierra, Juan José (2008): Humor y traducción. Los Simpson cruzan la frontera. Castelló: Publicacions de la Universitat Jaume I. ISBN: 978-84-8021636-4.

16. Ordóñez López, Pilar (2009): Miseria y esplendor de la traducción. La influencia de Ortega en la traductología. Castelló: Publicacions de la Universitat Jaume I. ISBN: 978-84-8021-691-3.

(No, la repetición del número 13 no es un error imputable al autor de este artículo, sino un lapsus en la numeración, ya que se asignó dicho número a dos volúmenes distintos y luego hubo que saltar el 14.)

Los dieciséis volúmenes que conforman la colección podrían clasificarse en las siguientes categorías:

a) volúmenes colectivos surgidos de jornadas y otros encuentros científicos (principalmente de las ya mencionadas jornadas sobre la traducción que periódicamente organiza el Departamento de Traducción y Comunicación de la UJI): 9 en total, concretamente los números 1, 2, 3, 5, 6, 7, 10, 12 y 13 (el primero de los dos 13); 
b) volúmenes colectivos no surgidos de ningún encuentro científico, sino concebidos de un modo más orgánico y escritos por encargo de los coordinadores: 2 en total, concretamente los números 9 y 11 ;

c) monografías: 4 en total, concretamente los números 8, 13 (el segundo de los dos 13), 15 y 16;

d) ediciones críticas: 1 , el número 4 .

En cuanto a la temática, como se puede ver, la colección cubre buena parte del espectro traductológico, ya que sus títulos abordan cuestiones que guardan relación con la teoría de la traducción (1 y 16), la práctica de la traducción (12), la epistemología de la traducción (8), la didáctica de la traducción (3 y 5), la historia de la traducción (9), la comunicación intercultural (11) y los distintos ámbitos de la traducción especializada: la literaria ( 2 y 13), la audiovisual (7 y 15), la científico-técnica (10) y la jurídica (13).

En lo que resta de este artículo se glosarán brevemente los contenidos y la estructura de cada uno de los volúmenes que componen la colección.

El número 1, cuyo título coincide con el nombre de la colección, es un compendio de reflexiones sobre la traducción que cubre gran parte de los enfoques teóricos en traductología. Baste mencionar, a título de ejemplo, la aportación de Ian Mason, que representa el enfoque textual, o la de Christiane Nord, en nombre del funcionalismo. A estos dos nombres extranjeros de sobra conocidos hay que sumar los de diversos especialistas españoles de prestigio, entre los cuales figuran la propia Amparo Hurtado, Roberto Mayoral, Rosa Rabadán, Julio César Santoyo o Mercedes Tricás.

El número 2, La traducció literària, recoge la mayor parte de las contribuciones a las segundas jornadas, que giraron en torno a este tema. Junto a los capítulos de Mary Snell-Hornby y José Lambert, más transversales, encontramos aportaciones centradas en cuestiones más concretas, como la traducción poética (Eustaquio Barjau), los elementos supuestamente intraducibles (Salvador Oliva), las cuestiones ideológicas (Francesc Parcerisas) o los problemas prácticos al traducir un relato determinado (Esther Benítez).

El número 3, La enseñanza de la traducción, recoge las contribuciones a las terceras jornadas y está estructurado en dos partes. La primera («La formación de traductores») contiene las conferencias de los especialistas invitados (Laura Berenguer, Amparo Hurtado, Allison Beeby, Pilar Elena, Christiane Nord, Basil Hatim, Leo Hickey,Natividad Gallardo y Teresa Cabré), mientras que la segunda («La formación de traductores en la Universitat Jaume I») incluye los planteamientos en cuanto a objetivos y metodología de un proyecto de investigación sobre didáctica de la traducción que se estaba llevando a cabo en aquel momento en el Departamento de Traducción y Comunicación de la UJI 
bajo la dirección de la profesora Hurtado. Esta segunda parte cubre buena parte de las materias que componen el currículo del estudiante de Traducción e Interpretación: las lenguas A, B y C, la traducción general, las traducciones especializadas (científico-técnica, jurídica, audiovisual y literaria) y la interpretación.

El número 4, Jerusalén, la emanación del gigante Albión, constituye claramente una excepción en el desarrollo de la colección, o, por lo menos, un camino que se abrió pero que no tuvo continuación, ya que es una edición crítica del texto de Blake mencionado en el título. El volumen incluye un estudio introductorio, la traducción del texto al español, aparato de notas y un glosario, todo ello a cargo de Xavier Campos Vilanova.

El número 5, Los estudios de traducción: un reto didáctico, fruto de las cuartas jornadas sobre la traducción de la UJI, es un retorno a las cuestiones didácticas y da fe del interés por estas cuestiones que sentía el colectivo que organizaba las jornadas y publicaba sus resultados. De nuevo, el abanico temático cubierto es amplio, pues incluye aspectos transversales (abordados por Jean Delisle, Michel Ballard, Sergio Viaggio, Antonio Argüeso, Roberto Mayoral y Marisa Presas) así como incursiones en la didáctica de la traducción especializada (Christine Durieux para la técnica y Holly Mikkelson para la jurídica).

El número 6, Cuerpos en serie, incluye diferentes aproximaciones a las relaciones entre dos conceptos que, en nuestra cultura, aparecen relacionados constantemente: la serialidad y la representación del cuerpo humano en los audiovisuales. Alrededor, pues, de este eje temático se van tejiendo distintas reflexiones, algunas de carácter netamente semiótico o fílmico, otras incluyendo la traducción entre sus preocupaciones. La segunda de las tres partes que conforman el libro («El texto audiovisual: traducción y representación») incluye aportaciones de Rosa Agost, Raquel Segovia, Encarna García Hinarejos y Jesús Molina.

El número 7, La traducción en los medios audiovisuales, recoge las contribuciones a las quintas jornadas, centradas en la traducción audiovisual, y se estructura en cinco grandes bloques: «Aspectos pragmáticos de la traducción audiovisual», «Aspectos metodológicos de la traducción audiovisual», «Aspectos profesionales», «Extranjerización o adaptación» y «La adaptación cinematográfica y dramática como traducción». Es imposible nombrar aquí a todos los autores que aportan contribuciones a este libro, pero podría quizá destacarse, sin menoscabo de los demás, a Ian Mason, Yves Gambier, JeanJacques Alcandre, Candace Whitman, Eliana Franco y Henrik Gottlieb entre los extranjeros, y a Roberto Mayoral, Patrick Zabalbeascoa, Frederic Chaume, Rosa Agost, Joan Fontcuberta, Laura Santamaría y Raquel Merino entre los españoles. 
El número 8, Aspectos epistemológicos de la traducción, del profesor Roberto Mayoral, constituye un punto de inflexión en la colección, ya que es la primera monografía que se publica en ella. Este libro somete a un escrutinio radical el carácter científico de los estudios sobre la traducción, y lo hace con rigor y sistematicidad y huyendo siempre de la autocomplacencia. Tras examinar las formas de conocimiento científico y los tipos de disciplinas, enumera una serie de criterios de cientificidad y los aplica a nuestra disciplina con el fin de comprobar en qué medida se cumplen.

El número 9, Essays on Medieval Translation in the Iberian Peninsula, abre también una nueva senda en la colección: la de los volúmenes colectivos que no son resultado de jornadas o encuentros científicos. El contenido es asimismo novedoso, ya que nunca con anterioridad se habían abordado cuestiones relacionadas con la historia de la traducción de modo monográfico. Las contribuciones al estudio de la traducción hacia las distintas lenguas peninsulares durante la Edad Media se deben principalmente a expertos en romanística, entre los que predominan los hispanistas y los catalanistas. Si bien la lengua vehicular del volumen, como se decía más arriba, es el inglés, la mayor parte de capítulos están escritos en español o en catalán, aunque hay también uno en francés y otro en italiano.

El número 10, La traducción cientifico-técnica y la terminología en la sociedad de la información, es resultado de las sextas jornadas sobre traducción celebradas en la UJI. El libro se estructura en cinco grandes bloques: «La comunicación multilingüe especializada», «Investigación sobre traducción científico-técnica y terminología en universidades españolas», «Perfiles profesionales de la traducción científico-técnica», «Desarrollo y utilización de herramientas de ayuda a la traducción» y «Programas de traducción asistida y bancos de datos terminológicos». No es posible aquí hacer justicia al elenco de especialistas que contribuyeron a este volumen, pero conviene destacar, entre los extranjeros, a Juan Carlos Sager y a Reiner Arntz, y, entre los procedentes de universidades españolas, a Ricardo Muñoz, Teresa Cabré, Anne Martin, el grupo PACTE, Gloria Corpas, Emilio Ortega, Pamela Faber y Natividad Gallardo. (De nuevo, perdón por las omisiones, únicamente justificadas por las restricciones de espacio.)

El número 11, Claves para la comunicación intercultural, del grupo CRIT (Comunicación y Relaciones Interculturales y Transculturales), es, al igual que el 9, un volumen colectivo pero no basado en ningún encuentro científico. Como se deduce del título, los contenidos de este libro son muy afines al campo de la traductología y se solapan en parte con él, pero sin coincidir del todo, ya que la comunicación intercultural no siempre tiene lugar a través de la traducción. El libro se divide en dos partes: «Planteamientos teóricos y metodológicos», con sendas contribuciones de Carlos Hernández y Francisco 
Raga, y «Análisis de interacciones comunicativas con inmigrantes», cuyos autores son Roberto Ortí, Dora Sales, Francisco Raga, Enric Sánchez y Mary Farrell. La primera parte es teórica, mientras que la segunda da cuenta de estudios empíricos.

El número 12, Experiencias de traducción. Reflexiones desde la práctica traductora, recoge la versión escrita de un conjunto de conferencias pronunciadas en el marco del Seminario Permanente del Departamento de Traducción y Comunicación entre 1997 y 2003. El propósito de dicho seminario, que sigue existiendo en la actualidad, es acercar a los estudiantes el mundo de la práctica profesional de la traducción y la interpretación. Así pues, en este volumen se da cobertura a un amplio abanico de ámbitos profesionales (como la traducción de series de televisión o la interpretación de lengua de signos, por poner dos ejemplos al azar) y de cuestiones más transversales dentro de la profesión (como la problemática del traductor autónomo o la direccionalidad). Entre los autores incluidos en este volumen figuran, aparte de las coordinadoras, Miguel Hagerty, Dorothy Kelly, Roberto Mayoral o Cristina Peri Rossi.

El número 13, La traducción y la interpretación en las relaciones jurídicas internacionales, es un compendio de las contribuciones a las séptimas jornadas, centradas en la traducción jurídica. El libro se articula en cuatro grandes bloques: «¿Qué es la traducción jurídica», «Retos académicos de la traducción jurídica: docencia e investigación», «Reflexiones desde la profesión» y «Colorear la literatura gris: últimas tesis en traducción jurídica». Como se ve, hay una cierta recurrencia en algunos de los volúmenes derivados de las jornadas por lo que se refiere a los criterios que guían la estructuración de los contenidos, ya que se suele partir de consideraciones teóricas (conceptuales, en este caso) para luego abordar los aspectos docentes, investigadores y profesionales del ámbito de especialidad del que se trate (aquí, el jurídico). Entre los autores que participaron en este volumen, podría destacarse a Leo Hickey, Jean-Claude Gémar y Luis Pérez entre los adscritos a universidades extranjeras, y, entre los procedentes de universidades españolas, a Roberto Mayoral, Emilio Ortega, Cristina Valderrey y Catherine Way (aparte de las propias coordinadoras del volumen, Anabel Borja y Esther Monzó).

El segundo número 13, El otoño del pingüino. Análisis descriptivo de la traducción de los culturemas, es, al igual que los dos siguientes, números 15 y 16, una monografía surgida de una tesis doctoral convenientemente reescrita y ajustada a las convenciones del formato libro, muy distinto del de una tesis. El volumen de Lucía Molina se estructura en tres partes: «Referentes teóricos», «Instrumentos de análisis para la traducción de los culturemas» y «Análisis de la traducción de los culturemas». En la tercera parte, que es la propiamente empírica, se analiza la traducción de los culturemas, o referentes culturales, en un corpus compuesto por tres traducciones al árabe de Cien años de soledad. 
El número 15, Humor y traducción. Los Simpson cruzan la frontera, es, como puede deducirse del título, un estudio de la traducción del humor en la serie de dibujos animados Los Simpson. Tras caracterizar la traducción audiovisual y el doblaje, y presentar su marco teórico y metodológico (que incide de manera especial en los aspectos pragmáticos y culturales), el autor procede a presentar los resultados del análisis del corpus, en términos tanto cuantitativos como cualitativos. Este trabajo contribuye sin duda a la dignificación académica de una modalidad de traducción (la audiovisual) y de un género (la serie de animación) que no han recibido la atención que merecen hasta hace bien poco tiempo.

Finalmente, el número 16, Miseria y esplendor de la traducción. La influencia de Ortega en la traductología, es al mismo tiempo un estudio sistemático y muy documentado del famoso ensayo de Ortega y Gasset y una reivindicación de su valor y su importancia en la reflexión traductológica contemporánea, así como de su influencia en otros pensadores.

Si, con la perspectiva que da el paso del tiempo, hacemos balance de lo conseguido por la colección «Estudis sobre la traducció» desde aquel ya remoto 1994, en el que vio la luz el primer volumen, hasta la actualidad, podría decirse que las luces predominan sobre las sombras. Parece indudable que en el seno de la colección se han publicado libros que podrían considerarse de referencia en el panorama bibliográfico de la traductología en general y, de modo más relevante, de sus respectivos ámbitos de especialidad. Sería buena prueba de ello la gran cantidad de citas de las que han sido objeto. El motivo de esta buena acogida de muchos libros de la colección y de su evidente impacto sobre estudios posteriores no es otro que la calidad de sus contenidos, que en muchísimas ocasiones han salido de la pluma de los máximos especialistas a escala internacional. Además, como ya se ha señalado más arriba, se ha conseguido cubrir muchas áreas temáticas de los estudios sobre la traducción, por lo que podríamos ahora añadir que la calidad no está reñida con la amplitud de espectro. Ahora bien, hay que reconocer que el punto débil de la colección las sombras- es el reducido número de títulos publicados, que genera, como consecuencia, una impresión de falta de continuidad. En efecto, 16 títulos en 17 años de andadura no es mucho, y hay aquí margen para la autocrítica. Este déficit de títulos podría explicarse en parte por la devaluación, ya señalada más arriba, de que son objeto las publicaciones que ven la luz en la misma universidad a la que está adscrito el autor / profesor, que ha llevado a muchos de los miembros del Departamento de Traducción y Comunicación de la Universitat Jaume I a publicar los resultados de su investigación en otras editoriales. Pero, en cualquier caso, se están explorando otras vías para nutrir de títulos a la colección. Baste decir que actualmente se encuentran en fase de preparación tres libros: uno de Óscar Díaz Fouces y Roberto Mayoral sobre 
traducción especializada (ya en imprenta), otro de Miguel Tolosa sobre el error en traducción y un tercero que sería una traducción al español del título ya clásico de Christiane Nord Text Analysis in Translation. Así pues, a pesar de la limitación que se acaba de reconocer, nuestra colección goza de un reconocimiento general y no existe ningún motivo para que no alcance una larga vida.

(Puede obtenerse información sobre todos los libros centrados en la traducción y la interpretación publicados por la Universitat Jaume I en la siguiente dirección:

http://www.tenda.uji.es/pls/iglu/!GCPPA00.GCPPR0006?lg=CA\&temaid=1 7.) 